\title{
ExamRandomizeR: Una aplicación web para la generación de exámenes aleatorizados que faciliten situar el Examen como herramienta de aprendizaje y no solo de evaluación en el aula de matemáticas ${ }^{*}$
}

\author{
Elvira Ferre Jaén ${ }^{1}$, Laura del Río Alonso ${ }^{1}$ y Antonio Maurandi López ${ }^{1}$
}

${ }^{1}$ Universidad de Murcia

\begin{abstract}
In this paper we present the development and usage of an interactive application for teaching mathematics, which facilitates the improvements of teaching and learning processes and it promotes the participation of students in the development of a dinamic class. The application has been programmed with $R$ and the package Shiny.

The application is able to generate, from a set of previously introduced questions, different randomized versions of the original exam, with their corresponding answers, thereby facilitating and automating the exam creation work.
\end{abstract}

Keywords: Evaluation, Didactics, Mathematics, R-project, Programming, Shiny, $R$

\section{Resumen}

En este trabajo se presenta el desarrollo y uso de una aplicación web interactiva como herramienta docente para la docencia de las matemáticas, que permite mejorar el proceso de enseñanza-aprendizaje del alumnado y desarrollar en el aula estilos educativos interactivos para fomentar la participación de los y las estudiantes. La aplicación ha sido programada con $R$ y la librería Shiny.

Esta aplicación es capaz de generar de forma aleatoria, a partir de una serie de preguntas introducidas previamente, exámenes aleatorizados, diferentes, junto con sus correspondientes soluciones, pudiendo asi facilitar y automatizar estas tareas.

Keywords: Evaluación, Didáctica, Matemáticas, R-project, Programación, Shiny, $R$

\footnotetext{
*Proyecto desarrollado con colaboración del Servicio de Apoyo a la Investigación (SAI) de la Universidad de Murcia
} 
ExamRandomizeR: Una aplicación web para la generación de exámenes aleatorizados que faciliten situar el Examen como herramienta de aprendizaje y no solo de evaluación en el aula de matemáticas

\section{Introducción}

A pesar de que la relevancia de la Didáctica de las Matemáticas en los estudios de Ciencias Sociales es incuestionable, es un hecho conocido que el aprendizaje de las matemáticas entraña una gran dificultad para estudiantes de titulaciones no técnicas. Esta dificultad, además, se constata en la enseñanza de las matemáticas en todos los niveles educativos.

En el ejercicio de la docencia, el examen se entiende y se utiliza como una herramienta cuyo único objetivo es el de evaluar el proceso de enseñanza aprendizaje y cuantificar el conocimiento adquirido por el alumnado al final del mismo.

Desde este punto de vista, el examen suele generar rechazo entre el alumnado y cierto grado de ansiedad (Lyons y Beilock 2012), perdiéndose de esta manera gran parte del potencial didáctico que esta herramienta puede ofrecer, aunque para ello sea necesario emplearlo con otros fines y otras metodologías, tal como propone el profesor Mazur (Mazur 2009).

Con esta premisa en mente, y con el objetivo último de crear en el aula un ambiente de aprendizaje dinámico e interactivo, donde el estudiantado pueda evaluar tanto como el equipo docente la adquisición de los contenidos que se trabajan en cada momento, hemos desarrollado con el lenguaje de programación R (R Core Team 2017) y la librería Shiny (Chang y col. 2016), una aplicación web interactiva de sencillo manejo, que puede ser utilizada tanto por docentes como por discentes.

La aplicación permite, a partir de un examen base que contendrá un listado de preguntas de tipo uniforme, como puede ser, el de respuesta múltiple, generar diversos exámenes o modelos de examen en los que se reordenan de forma aleatoria tanto las preguntas como las respuestas, utilizando semillas de aleatorización, lo que permite volver a generar los mismos exámenes si fuera preciso.

La herramienta se presenta como una aplicación de acceso web que devuelve los resultados aleatorizados tanto en ficheros tex de $\mathrm{LAT}_{\mathrm{E} X}$ (Project 2017), Markdown (Gruber 2017), formato txt de texto plano (ASCII) o directamente en pdf.

Existen, aparte de esta aplicación, otras herramientas interesantes y bastante completas de creación de exámenes como son: el paquete exams de R (Grün y Zeileis 2009), esta herramienta, aunque es muy completa, no se centra en los mismos objetivos que la nuestra y adolece de una interface web de fácil uso para usarla sin conocimientos de $\mathrm{R}$, no obstante se trata de una potente herramienta para generar exámenes dinámicos que pueden ser diferentes para cada persona y dispone de una licencia GNU General Public Licence. Otra opción es "Schoolhouse Test 4" (School Housetest Tecnologies 2017) diseñado expresamente para docentes. Schoolhouse Test 4 te permite crear exámenes con muchas opciones, incluyendo aleatorización de preguntas y dispone de un modulo para matemáticas. No obstante se trata de software privativo, hay que adquirir una licencia y solo está disponible para el sistema operativo Windows.

Se pueden encontrar otras herramientas web para crear formularios para ser contestados on-line, algunas de estas te permiten aleatorizar ítems como es "Qualtrics 360 plataform" (Qualtrics 360 platform 2017), aunque esta no tiene una licencia que permita su uso sin un contrato de servicios. Otro buen ejemplo de cuestionarios on-line lo constituye la aplicación Encuestas de la Universidad de Murcia (Web de ENCUES- 
TAS Version 2.5). Ninguna de estas últimas te permite generar exámenes para ser distribuidos en papel, circunscribiéndose a cuestionarios on-line si prestar atención a la presentación de formulas matemáticas. De esta forma pensamos que nuestro desarrollo es pertinente y pone a disposición de docentes y discentes una interesante opción para crear modelos de exámenes con las particularidades que pasamos a especificar.

\section{Objetivos}

Los objetivos de este trabajo son los siguientes:

1. Crear una aplicación que sea capaz de generar exámenes partiendo de un examen inicial, por medio de permutaciones aleatorizadas de las preguntas y de las respuestas de cada pregunta.

2. Que la aplicación permita la introducción de formulas matemáticas complejas en los exámenes sin que ello genere errores en el formato final.

3. Que la aplicación permita crear claves/plantillas de corrección para cada uno de los exámenes generados.

4. Que los formatos de salida sean variados y permitan la interacción de docentes y discentes: $\mathrm{LT}_{\mathrm{E}} \mathrm{X}$, Markdown, txt, pdf.

5. Que la aplicación tenga un diseño cómodo para su uso de forma intuitiva y con acceso vía web.

\section{Desarrollo de la innovación}

Se plantearon cuatro fases en el desarrollo de la aplicación:

1. Una primera fase donde nos centramos en el desarrollo del algoritmo de randomización. Este no es complejo y no ocupa más de 150 lineas de código, pero ha de ser robusto y de fácil mantenimiento.

2. Una segunda fase de diseño de la aplicación web con un o dos formatos de salida a fin de poder ponerla en semiproducción.

3. Una tercera etapa de prueba en la que diferentes docentes del área de Didáctica de las Ciencias Matemáticas la probaron en el desarrollo de su clases y en los exámenes de las diferentes asignaturas del área. Muchas de las sugerencias de estos docentes fueros incluidas como mejoras a la aplicación y otras aún están pendientes de ser implementadas.

4. Una cuarta etapa de mejora de los formatos de salida para adaptarla a las diversas necesidades y formas de aplicación.

5. Una quinta etapa de mejora de los formatos de carga de datos para por ejemplo poder leer directamente de una plantilla escrita en Markdown

6. Una última etapa de adaptación de los css de la aplicación a los colores institucionales. 
ExamRandomizeR: Una aplicación web para la generación de exámenes aleatorizados que faciliten situar el Examen como herramienta de aprendizaje y no solo de evaluación en el aula de matemáticas

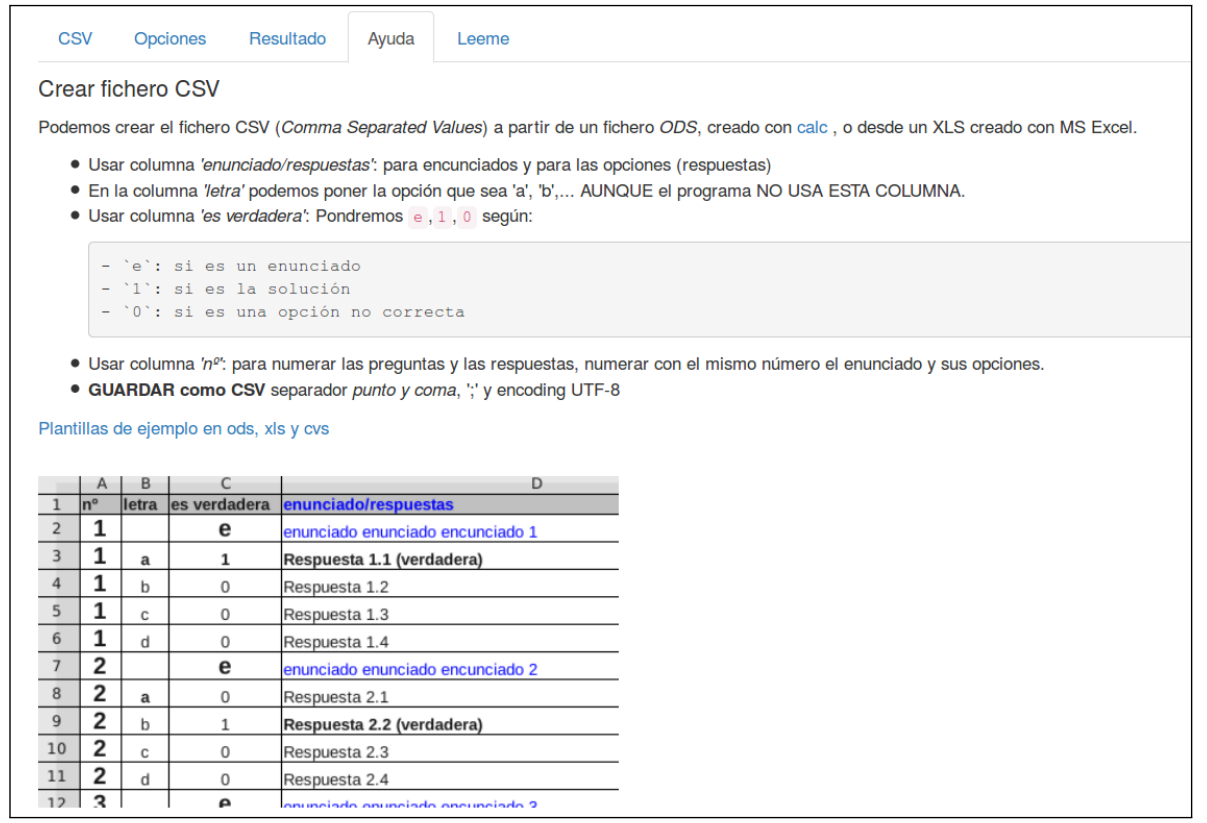

Fig. 1: Aspecto de la hoja de cálculo modelo para la creación de exámenes.

Actualmente nos encontramos en la última fase del proyecto.

\section{Resultados}

La aplicación en su actual versión 3.1.4 puede ser accedida y utilizada desde la url: http://gauss.inf.um.es:8080/examrandomizer/. Hasta la fecha ha sido empleada con asiduidad durante los cursos de 2015/2016 y 2016/2017 con gran aceptación por parte del profesorado de las asignaturas de Matemáticas y su Didáctica I y II , del segundo y tercer curso del Grado en Educación Primaria de la Universidad de Murcia.

El empleo de la aplicación requiere de cuatro fases:

1. Carga de datos

2. Especificación de opciones para la creación de los exámenes

3. Selección del formato de salida y creación de exámenes

4. Descarga y edición de los modelos de examen

En la fase de "carga de datos", hay que subir el modelo de examen base en un formato csv que se explica con detalle en el apartado ayuda, ver Figuras 1 y 2. El examen base debe contener los enunciados de las preguntas y las posibles respuestas, indicando con un código cual de las preguntas es la correcta. Se facilita una hoja de cálculo como ejemplo que sirve de base para crear un examen y adquirir la rutina de carga de datos.

En la fase de "especificación de opciones para la creación de los exámenes", debemos ser consistentes en la forma de las preguntas, estas deben de ser, para estas primeras versiones de la aplicación, todas del mismo tipo, es decir, si optamos por cuatro 


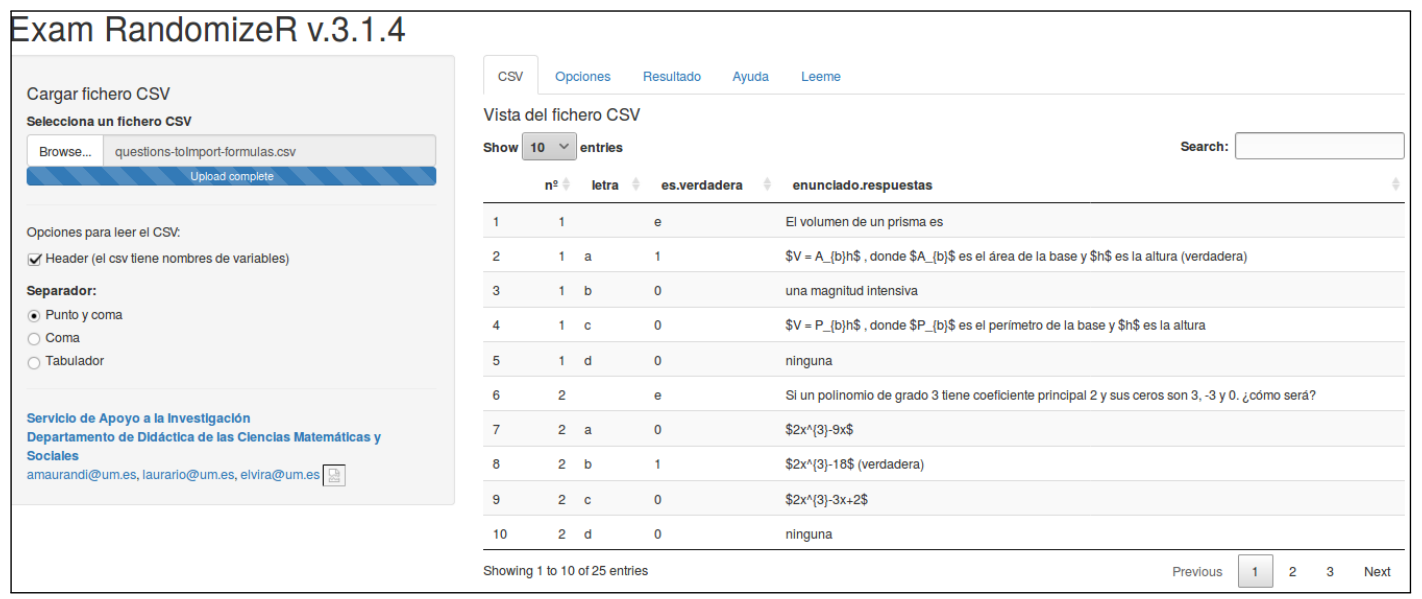

Fig. 2: Carga de datos en formato csv.

respuestas posibles con una sola respuesta válida, todas las preguntas deberán de ser de ese formato. También deberemos especificar el número de exámenes o modelos que deseamos generar, y si queremos aleatorizar el orden de las preguntas, las respuestas de cada pregunta o ambas cosas, ver Figura 3 y 4.

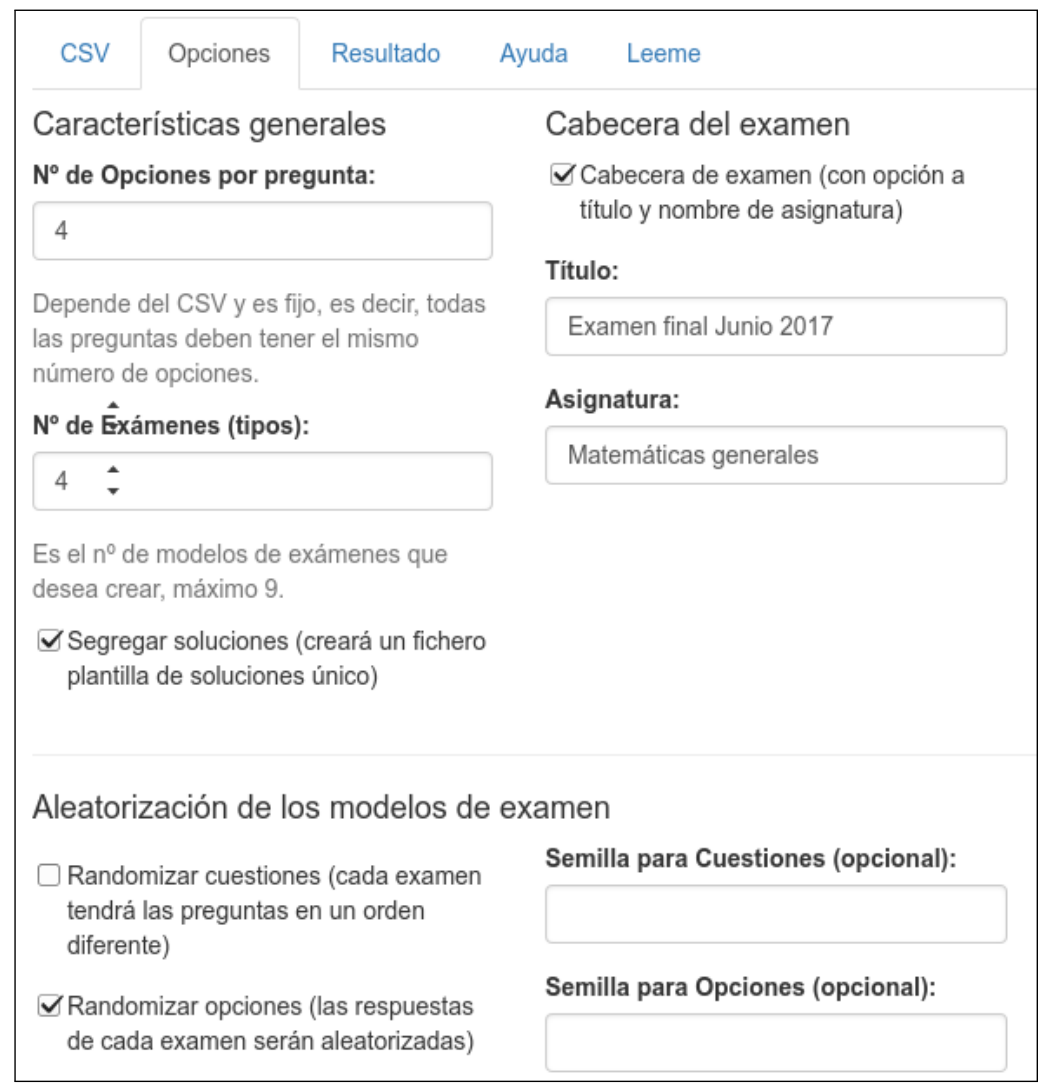

Fig. 3: Opciones de creación de exámenes. 
ExamRandomizeR: Una aplicación web para la generación de exámenes aleatorizados que faciliten situar el Examen como herramienta de aprendizaje y no solo de evaluación en el aula de matemáticas

"Aleatorización de modelos de examen". La posibilidad de desvincular la aleatorización de las preguntas y las respuestas permite presentar varios modelos del mismo examen donde el orden de las preguntas es el mismo, pero el orden de las respuestas es diferente. Todas estas herramientas permiten crear múltiples "exámenes", y fomentar que, aunque alguna pregunta haya aparecido en otras ocasiones, contestar el examen requiera volver a razonar y pensar la respuesta que se da. Una utilidad que surge de esta posibilidad es que, el hecho de disponer de numerosos modelos para exámenes, desanima al alumnado a intentar copiar de los compañeros, pues se incrementa la dificultad de comunicación y se estimula que se centren en la resolución del propio examen.

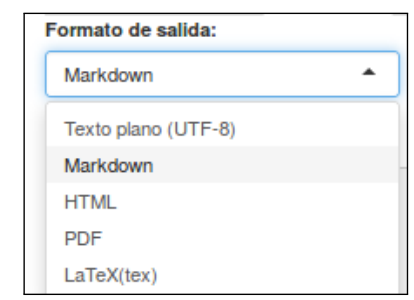

Fig. 4: Opciones: Formatos de salida.

La etapa, "descarga y edición", actualmente está implementada para obtener los exámenes en html. Markdown, txt, tex y pdf, de forma que es sencillo incluir las preguntas tipo test en un examen más general o generar exámenes solo de preguntas tipo test. Otros formatos de salida, como por ejemplo docx están siendo desarrollados en estos momentos.

La última etapa, "descarga y edición", actualmente está implementada para obtener los exámenes en Markdown y txt, de forma que es sencillo incluir las preguntas tipo test en un documento de examen más general o generar exámenes solo de preguntas tipo test, el resto de formatos mencionados están siendo desarrollados en estos momentos.

\section{Conclusiones}

Nuestro objetivo final es implementar en el aula un proyecto basado en lo que se conoce como Interactive teaching, cuya premisa es que la aplicación práctica capacita a los y las estudiantes a comprender la profundidad de los materiales de estudio (Yakovleva y Yakovlev 2014).

Estos estilos de aprendizaje interactivo proporcionan cuatro formas básicas de retroalimentación:

- Los logros de los y las estudiantes son medibles, el profesorado que hace uso de estilos de enseñanza interactivos está mejor preparado para conocer cómo dominan sus estudiantes un material determinado.

- Flexibilidad en la enseñanza, este tipo de metodología involucra comunicaciones bidireccionales permiten al docente hacer ajustes rápidos en los procesos y enfoques de enseñanza.

- La práctica hace la perfección. La instrucción interactiva mejora el proceso de aprendizaje. 
- La motivación del estudiante. La enseñanza de dos vías disipa la pasividad del estudiante

En este sentido, la aplicación "ExamRandomizeR", aunque se encuentra en un estado avanzado de desarrollo, todavía estamos progresando en otros aspectos fundamentales como los formatos de salida, especialmente en el empleo de plantillas avanzadas de IATEX. La ventaja de generar salidas directamente a Markdown nos permitirá aprovechar el potencial de este lenguaje para crear formato html fácilmente y por lo tanto nos permite crear herramientas de evaluación en web. Este hito es importante, pues las herramientas on-line son esenciales si queremos aplicar la estrategia de aprendizaje interactivo sugerida por el profesor Mazur.

Pretendemos, por otro lado, aparcar el modelo clásico de profesor o profesora que resuelve problemas en clase, y plantear en su lugar sesiones interactivas en las que los y las estudiantes aprendan preguntando y resolviendo aquellas cuestiones generadas por el grupo, razonando y generando debate en clase, relegando el estilo clásico de aprendizaje "memorizando recetas".

Para resolver durante las sesiones prácticas las cuestiones que le surgen al alumnado tras el uso de ExamRandomizeR, pretendemos seguir el siguiente esquema (Mazur 2009):

1. Planteamiento de las preguntas

2. Tiempo para pensar en la respuesta (2 minutos)

3. Respuesta individual de cada alumno o alumna (levantar manos y recuento de respuestas)

4. Planteamos un debate y dejamos tiempo para que comuniquen su razonamiento a los compañeros

5. Responden otra vez

6. Explicación de la respuesta correcta por parte del docente

Mediante este proceso, al hacer que el alumnado discuta, el foco de la información se desplaza del o la docente, y se centra sobre el alumnado, permitiéndoles razonar sobre los conceptos planteados y ver dónde se encuentran con respecto al resto de la clase, sin que eso afecte a su nota final, por lo que este sistema se convierte en un tipo de evaluación informativa que se lleva a cabo de manera continua.

Nos basamos en el hecho de que si los y las estudiantes comprenden los conceptos básicos, si son capaces de contestar correctamente los cuestionarios generados aleatoriamente con ExamRandomizeR podrán resolver correctamente los problemas convencionales.

En resumen, planteamos que el alumnado necesita adquirir una comprensión razonada de la información que reciben, siendo de limitada utilidad la mera memorización de ecuaciones o teorías matemáticas, si no va acompañado de la comprensión y razonamiento de esas teorías mediante su uso. En definitiva, en la enseñanza de las matemáticas es necesario trabajar en la construcción de modelos mentales para que se asimile la información presentada en clase. 
ExamRandomizeR: Una aplicación web para la generación de exámenes aleatorizados que faciliten situar el Examen como herramienta de aprendizaje y no solo de evaluación en el aula de matemáticas

\section{Ejemplo completo de utilización}

Comenzamos creando una serie de preguntas de múltiple respuesta donde solo una es correcta, lo hacemos con la aplicación Calc de la suite ofimática LibreOffice.

\begin{tabular}{|c|c|}
\hline $\mathrm{n}^{\circ}$ letra & es verdadera enunciado/respuestas \\
\hline 1 & El volumen de un prisma es \\
\hline $1 \mathrm{a}$ & $\left.1 \$ \mathrm{~V}=\mathrm{A} \_\mathrm{b}\right\} \mathrm{h} \$$, donde $\left.\$ \mathrm{~A} \_\mathrm{b}\right\} \mathrm{b}$ es el área de la base y $\$ \mathrm{~h} \$$ es la altura (verdadera) \\
\hline $1 \mathrm{~b}$ & 0 una magnitud intensiva \\
\hline $1 \mathrm{c}$ & $0 \$ \mathrm{~V}=\mathrm{P}\{\mathrm{b}\} \mathrm{h} \$$, donde $\$ \mathrm{P}\{\mathrm{b}\} \$$ es el perimetro de la base y $\$ \mathrm{~h} \$$ es la altura \\
\hline $1 \mathrm{~d}$ & 0 ninguna \\
\hline 2 & Si un polinomio de grado 3 tiene coeficiente principal 2 y sus ceros son $3,-3$ y 0 . ¿cómo será? \\
\hline $2 \mathrm{a}$ & $0 \$ 2 x^{\wedge}\{3\}-9 x \$$ \\
\hline $2 \mathrm{~b}$ & $1 \$ 2 x^{\wedge}\{3\}-18 \$$ (verdadera) \\
\hline $2 \mathrm{c}$ & $0 \$ 2 x \wedge\{3\}-3 x+2 \$$ \\
\hline $2 \mathrm{~d}$ & O ninguna \\
\hline 3 & ¿Qué nombre recibe la diferencia entre los valores extremos de una variable estadística? \\
\hline 3a & OMediana \\
\hline $3 \mathrm{~b}$ & OModa \\
\hline $3 \mathrm{c}$ & 1 Recorrido (verdadera) \\
\hline $3 \hat{d}$ & 0 ninguna \\
\hline
\end{tabular}

Fig. 5: Creación de examen con Calc de LibreOffice.

Nuestro propósito en este ejemplo es crear varios modelos de examen, ya que tenemos un grupo muy numeroso, vamos a crear 4 grupos, y podemos seleccionar si aleatorizar el orden de las preguntas y el orden de las respuestas, solo el orden de las preguntas o solo el de las respuestas, está ultima opción produce exámenes que se parecen, pues las preguntas son las mismas pero presentan las respuestas en diferente orden. Gracias a la plantilla de soluciones podremos corregir los exámenes sin confundirnos a pesar de ser muy parecidos.

Como se puede ver en la figura 3 , seleccionamos las opciones sobre el formato: $\mathrm{N}^{\circ}$ de Opciones por pregunta igual a $4, \mathrm{~N}^{\circ}$ de exámenes (tipos) a crear, también igual a 4. Verificamos que está seleccionado Segregar soluciones para crear un fichero independiente con las respuestas. Seleccionamos Cabecera de Examen para editar el Titulo y la Asignatura. En Aleatorización de los modelos de examen seleccionamos Randomizar opciones.

Un resumen del proceso de creación de exámenes se puede ver en la pestaña resultados" de la aplicación, ver figura 6, junto con el aspecto de los ficheros después de la descarga. El examen creado contendrá el Titulo y Asignatura que consignáramos en Opciones, un ejemplo se puede ver en la figura 7. 


\begin{tabular}{|c|c|c|c|c|c|c|c|c|}
\hline CSV & \multicolumn{2}{|c|}{ Opciones } & \multicolumn{2}{|c|}{ Resultado } & \multicolumn{2}{|c|}{ Ayuda } & \multicolumn{2}{|c|}{ Leeme } \\
\hline \multicolumn{7}{|c|}{ Formato de salida: } & \multirow{2}{*}{\multicolumn{2}{|c|}{$\begin{array}{l}10 \text { soluciones.pdf } \\
\text { soluciones.md }\end{array}$}} \\
\hline PDF & & & 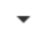 & & & & & \\
\hline \multicolumn{7}{|c|}{ Opciones seleccionadas } & \multicolumn{2}{|c|}{$\begin{array}{l}11 \text { examen-Norandomizado-4.pdf } \\
\text { examen-Norandomizado-4.md }\end{array}$} \\
\hline \multicolumn{7}{|c|}{$\begin{array}{l}\text { Opciones que usted seleccionó: } \\
\text { № de Respuestas:4 } \\
\text { № de Examenes:4 } \\
\text {-Soluciones Segregadas } \\
\text { - Cuestiones NO Randomizadas } \\
\text { - Opciones Randomizadas. } \\
\text {-Examenes con Cabecera } \\
\text {-Titulo: Examen final Junio } 2017 \\
\text {-Asignatura: Matemáticas generales } \\
\text { Formato de salida: PDF }\end{array}$} & \multicolumn{2}{|c|}{$\begin{array}{l}\text { examen-Norandomizado-3.pdf } \\
\text { examen-Norandomizado-3.md } \\
\text { examen-Norandomizado-2.pdf } \\
\text { examen-Norandomizado-2.md } \\
\text { examen-Norandomizado-1.pdf } \\
\text { examen-Norandomizado-1.md }\end{array}$} \\
\hline \multicolumn{9}{|c|}{ Crear exámenes } \\
\hline \multirow{2}{*}{\multicolumn{9}{|c|}{$\begin{array}{l}\text { Si ya ha subido un CSV y seleccionado las opciones 'presione } \\
\text { Resultados de la creación de exámenes }\end{array}$}} \\
\hline & & & & & & & & \\
\hline Orden & Q1 & s1 & Q2 & s2 & Q3 & s3 & Q4 & S4 \\
\hline 1 & 1 & $b$ & 1 & a & 1 & a & 1 & a \\
\hline 2 & 2 & d & 2 & c & 2 & d & 2 & a \\
\hline 3 & 3 & a & 3 & a & 3 & c & 3 & d \\
\hline 4 & 4 & a & 4 & c & 4 & c & 4 & d \\
\hline 5 & 5 & b & 5 & a & 5 & a & 5 & d \\
\hline \pm Desc & gar re & sutan & s en u & n zip & & & & \\
\hline
\end{tabular}

Fig. 6: Resultado de la creación de exámenes en formato pdf.

\section{Examen final Junio 2017}

Matemáticas generales

Modelo de Examen $n^{\circ}: 3$

- Estudiante(nombre completo):

- DNI:

- Curso y grupo:

- Fecha:

1. El volumen de un prisma es

a. $V=A_{b} h$, donde $A_{b}$ es el área de la base y $h$ es la altura (verdadera)

b. ninguna

c. $V=P_{b} h$, donde $P_{b}$ es el perímetro de la base y $h$ es la altura

d. una magnitud intensiva

2. Si un polinomio de grado 3 tiene coeficiente principal 2 y sus ceros son $3,-3$ y 0 . ¿cómo será?
a. ninguna
b. $2 x^{3}-3 x+2$
c. $2 x^{3}-9 x$
d. $2 x^{3}-18$ (verdadera)

Fig. 7: Vista de uno de los modelos de examen creados en formato pdf. 
ExamRandomizeR: Una aplicación web para la generación de exámenes aleatorizados que faciliten situar el Examen como herramienta de aprendizaje y no solo de evaluación en el aula de matemáticas

\section{Referencias bibliográficas}

ATICA, Universidad de Murcia. Web de ENCUESTAS Version 2.5. https: //encuestas . um.es.

Chang, Winston y col. (2016). shiny: Web Application Framework for R. R package version 0.14 .2 .

Gruber, John (2017). Daring Fireball. [Web; accedido el 31-03-2017].

Grün, Bettina y Achim Zeileis (2009). "Automatic Generation of Exams in R". En: Journal of Statistical Software 29.10, págs. 1-14. DOI: 10.18637/jss.v029.i10.

Lyons, Ian M y Sian L Beilock (2012). "When math hurts: math anxiety predicts pain network activation in anticipation of doing math". En: PloS one 7.10, e48076.

Mazur, Eric (2009). Confessions of a converted lecturer. Youtube. URL: https : / / youtu.be/WwslBPj8GgI.

Project, LaTeX (2017). The LaTeX Project. [Web; accedido el 31-03-2017].

Qualtrics 360 platform (2017). Question Randomization. https://www.qualtrics . com/support / employee-insights / onboarding / 360-onboarding/gettingstarted/. [Accedido 27-May-2017].

R Core Team (2017). R: A Language and Environment for Statistical Computing. R Foundation for Statistical Computing. Vienna, Austria.

School Housetest Tecnologies (2017). Schoolhouse Test 4. http://help. schoolhousetech. com/category/6-schoolhouse-test. [Accedido 27-May-2017].

Yakovleva, Nadezhda O y Evgeny V Yakovlev (2014). "Interactive teaching methods in contemporary higher education". En: Pacific Science Review 16.2, págs. 75-80. 